\title{
Central Pontine Myelinolysis in a Normonatremic Patient with Depression
}

\author{
Yu Chia Liu, ${ }^{1,2}$, Yen Kuang Yang ${ }^{1,3,4}$, Po See Chen ${ }^{1,3,5}$, Wei Hung Chang ${ }^{1,5}$ \\ ${ }^{1}$ Department of Psychiatry, National Cheng Kung University Hospital, College of Medicine, National Cheng Kung University, ${ }^{2}$ Department of \\ Psychiatry, Tainan Municipal Hospital (Managed by Show Chwan Medical Care Corporation), ${ }^{3}$ Institute of Behavioral Medicine, College of \\ Medicine, National Cheng Kung University, ${ }^{4}$ Department of Psychiatry, Tainan Hospital, Ministry of Health and Welfare, Tainan, ${ }^{5}$ Department \\ of Psychiatry, National Cheng Kung University Hospital, Dou-Liou Branch, Yunlin, Taiwan
}

\begin{abstract}
A 76-year-old male presented with a recurrent depressive episode, an unsteady gait and cognitive impairment. Extensive blood tests, including hemogram, biochemical tests, folic acid, vitamin B12, and thyroid hormone, showed normal results. With the exception of the unsteady gait, neurological examination was negative. Brian magnetic resonance imaging (MRI) showed the typical feature of central pontine myelinolysis (CPM); however, there was no history of alcoholism, liver transplantation, malnutrition or rapid correction of hyponatremia. The patient had taken venlafaxine to treat major depressive disorder for more than 20 years. After discontinuation of venlafaxine, the unsteady gait gradually resolved, and subsequent MRI revealed reduction of the lesions over 6 months. We discuss herein the possible correlation between chronic use of venlafaxine and CPM.
\end{abstract}

KEY WORDS: Myelinolysis, central pontine; Normonatremia; Venlafaxine hydrochloride.

\section{INTRODUCTION}

Central pontine myelinolysis (CPM) is a neurologic disorder characterized by symmetrical and non-inflammatory demyelination within the central basis pons. According to the study by Lampl and Yazdi [1], chronic alcoholism is the most common underlying disease correlated with CPM, the second and third most common being rapid correction of hyponatremia and liver transplantation. In addition, although relatively less common, CPM may occur in patients with burns, malnutrition, diabetes mellitus, brain tumor/cerebral edema, leukemia, chemotherapy, acquired immune deficiency syndrome, Wernicke's encephalopathy, Wilson's disease, adrenal insufficiency, renal disease, sepsis, sickle cell disease, and hyperemesis gravidarum $[1,2]$. The clinical manifes-

Received: October 25, 2020 / Revised: December 7, 2020

Accepted: December 15, 2020

Address for correspondence: Wei Hung Chang

Department of Psychiatry, National Cheng Kung University

Hospital, 138 Sheng Li Road, North Dist., Tainan 70403, Taiwan

E-mail: weihung2364009@gmail.com

ORCID: https://orcid.org/0000-0002-5964-106X tations of CPM include dysphagia, dysarthria, quadriparesis, movement disorders, seizures, lethargy, confusion, and coma [3]. However, few reports have described neuropsychiatric symptoms in CPM. In a review by Lampl and Yazdi [1], neuropsychiatric manifestations of CPM were reported to include cognitive dysfunction (deficits in attention, concentration, short-term memory, visual motor and fine motor speeds and learning ability), a pseudobulbar state with pathological laughing and crying, and even catatonia. The etiology and pathogenesis of CPM is still obscure. Herein, we describe a case of CPM without typical risk factors and symptoms.

\section{CASE}

This study was approved by the ethics committee of National Cheng Kung University Hospital (No. A-EC-109019). A written informed consent was obtained from the patient for the publication of this case report. A 76-yearold male was evaluated in the geriatric ward due to syncope and depression with suicidal ideation. He had received a diagnosis of major depressive disorder from a

(ㄷ) This is an Open-Access article distributed under the terms of the Creative Commons Attribution Non-Commercial License (http://creativecommons.org/licenses/by-nc/4.0) which permits unrestricted non-commercial use, distribution, and reproduction in any medium, provided the original work is properly cited. 
psychiatrist when he divorced at 26 years of age, after which he suffered depressive episodes related to psychosocial stressors. Trials of fluoxetine, sertraline, mirtazapine, and trazodone were ineffective, but the patient's depression diminished with the use of venlafaxine $150 \mathrm{mg}$, and he continued to use venlafaxine to control his depression for more than 20 years. Other notable medical history included hypertension, hyperlipidemia, coronary artery disease, and benign prostate hyperplasia. The patient took bisoprolol $5 \mathrm{mg}$, atorvastatin $20 \mathrm{mg}$, clopidogrel 75 $\mathrm{mg}$, and silodosin $4 \mathrm{mg}$ per day. His clinical history and laboratory findings excluded alcohol abuse, malnutrition, and electrolyte imbalance. The patient had three master's degrees and had worked as a scientific editor for more than 10 years.

Six months ago, the patient complained of frequent dizziness with near-syncope. Holter monitoring for 24 hours was performed to assess control of heart rate, and showed an average heart rate of 73 beats per minute (bpm), a maximum heart rate of $118 \mathrm{bpm}$, and a minimal heart rate of 50 bpm; no episodes of arrhythmia were detected. The tilt table test showed negative findings. At the same time, the patient reported recurrent depression, with depressed mood, loss of interest, fatigue, poor appetite, insomnia, poor attention, feeling of helplessness, and suicidal ideation after he deleted an important document on his computer. In addition, he misplaced his belongings fre- quently, and had difficulty in using the internet and smart phones prior to this depressive episode. He also complained of short-term memory impairment.

During hospitalization, extensive blood tests were carried out, and the results were all within normal limits, including hemogram, renal function, liver function, electrolytes (sodium, potassium, calcium, magnesium), folic acid, vitamin B12, and thyroid hormone measurements. The cranial nerve and upper and lower limb function were normal on neurological examination, with the exception of the unsteady gait. Cranial magnetic resonance imaging (MRI) showed (i) nodules with hyperintensity on T2 fluid-attenuated inversion recovery and hypointensity on diffusion-weighted images in the bilateral basal ganglia, left thalamus, bilateral cerebellum, periventricular and subcortical white matter, which were suspected to be related to an old infarction; (ii) symmetric hyperintensity on T2 imaging in the pons, which is typical of CPM (Fig. 1A); and (iii) generalized cerebral atrophy. Carotid artery sonography revealed moderate to severe carotid atherosclerosis of the right internal carotid artery with significant hemodynamic change. Thus, stent placement and angioplasty were performed.

After discharge, there was no obvious near-syncope episode, but the patient still had an unsteady gait, depressive symptoms, and poor cognitive function. Thus, psychological assessment was arranged for cognitive evaluation.
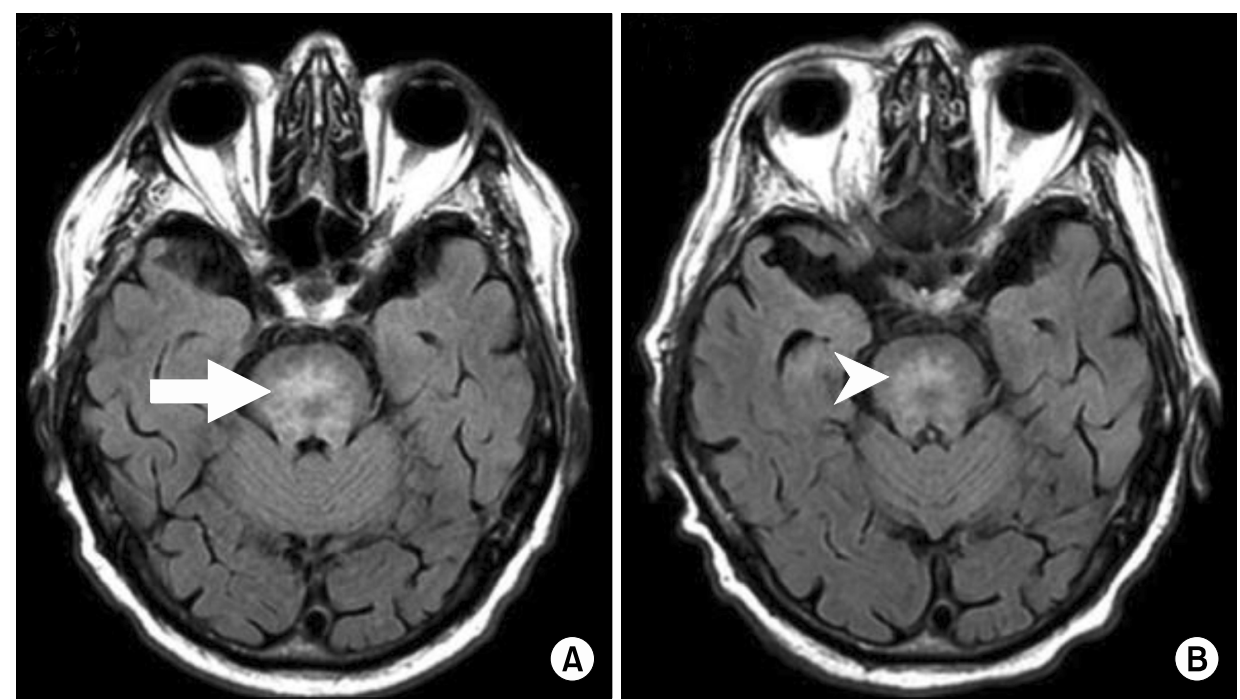

Fig. 1. Axial T2 fluid-attenuated inversion recovery image on magnetic resonance imaging scan. (A) Image showing a symmetrical area of hyperintensity in the basis points (arrow indicates site of lesion). (B) Image obtained 6 months later showing the decreased signal intensity of the lesion within the pons (arrowhead indicates site of lesion). 
The Wechsler adult intelligence scale (third edition) revealed the following: full-scale intelligence quotient (IQ): 127; verbal IQ: 139 (Verbal Comprehension Index: 148, Working Memory Index: 115), performance IQ: 107 (Perceptual Organization Index: 118, Processing Speed Index: 79). These results indicated that the patient's processing speed was below average as compared with the other cognitive domains. The Wisconsin Card-Sorting Test showed that the patient's ability to utilize environmental feedback to shift cognitive sets was below average (perseveration errors: 35, percentile score: 19 and number of categories completed: 1 , percentile score: $>16$ ). Because venlafaxine was ineffective for depression at this time, various other medications, including aripiprazole (5 $\mathrm{mg}$ per day for 2 weeks), mirtazapine ( $15 \mathrm{mg}$ per day for 3 weeks), lithium (300 mg per day for 8 weeks), and lurasidone (40 mg per day for 8 weeks), were administered, but failed to have an effect. The patient subsequently began treatment with agomelatine and quetiapine, and partial improvement of depressive symptoms was noted. Six months later, the patient was able to walk slowly without assistance. Subsequent brain imaging and cognitive evaluation were performed six months later, and the second cranial MRI showed a reduction in the area of hyperintensity on T2 imaging in the pons (Fig. 1B). A cognitive abilities screening instrument revealed definite neurocognitive deficit (total score: 83, cut-off point: 87), especially in orientation and animal-name fluency.

\section{DISCUSSION}

In our patient, a neurological symptom of CPM may have been an unsteady gait, as when the unsteady gait improved, a simultaneous reduction in the area of hyperintensity on T2 imaging in the pons was noted. Initial cognitive testing showed deficits in processing speed and executive function, but the tests were performed during a depressive episode, and so the results may have been influenced by depression [4]. Although signs of cognitive dysfunction were present prior to depression, there was no definite evidence to indicate that the cognitive dysfunction was associated with CPM.

The pathogenesis of CPM is not completely understood. Although CPM is usually associated with rapid correction of hyponatremia, it has also occurred in normonatremic $[5,6]$ and even hypernatremic patients [7]. Thus, a rapid increase in sodium concentration is not likely to be the only cause of CPM. Shah et al. [8] suggested that CPM may develop in susceptible patients with hypertonic stress, which may result from the correction or development of an electrolyte imbalance or disturbance in tonicity. Susceptible patients are those who have alcoholism, liver disease/orthotopic liver transplantation, malnutrition, malignancy, pregnancy/postpartum state, severe illness/sepsis, adrenal insufficiency, and metabolic derangements (hypoglycemia, hypokalemia, hypophosphatemia). In our patient, there were none of the potential risk factors mentioned above. In addition, after venlafaxine was discontinued, we prescribed various medications for different weeks, including aripiprazole, mirtazapine, lithium, and lurasidone, but there was no obvious effect. Finally, agomelatine and quetiapine were administered and the depressive symptoms were improved. About six months later, the patient's gait became steady, and the area of hyperintensity on $\mathrm{T} 2$ imaging in the pons decreased. Thus, pharmacological factors should be considered as a possible etiology of CPM in our case and venlafaxine is the most likely factor according to the sequence of medication. Most antidepressants have been noted to be associated with hyponatremia, especially selective serotonin reuptake inhibitors and venlafaxine, and the most likely mechanism of this adverse effect is the syndrome of inappropriate secretion of antidiuretic hormone (SIADH) [9] . This means that antidepressants may change tonicity by regulating antidiuretic hormones. We searched published articles that reported antidepressant-related CPM without other risk factors, and found only two related reports. Twardowschy et al. [10] described a 53-year-old female with fluoxetine-induced hyponatremia after fluoxetine had been administered for 9 days. After hyponatremia correction, the patient exhibited cognitive deficits in language, memory, and psychomotor retardation. Finally, pontine and extrapontine osmotic myelinolysis were diagnosed. In the report of Lupato et al. [11], a 69-year-old male was diagnosed with asymptomatic CPM, the possible etiology of which was chronic use of clomipramine (for about 10 years) and exposure to glue and chemical agents. The latter patient was relatively more similar to our case than the former, because we did not find any episode of hyponatremia or other known risk factors. Lupato et al. [11] also assumed that the pathogenesis of CPM could be a direct myelion-toxic mechanism or osmotic 
damage secondary to antidepressant-induced asymptomatic SIADH. Thus, we speculated that a similar osmotic injury that causes the myelinolytic process is associated with chronic use of specific antidepressants.

In conclusion, we described a case of CPM in which no hyponatremia was present. We hypothesized that patients who use antidepressants may be susceptible to disturbance in tonicity. This case also implied that when patients using antidepressants have new onset of neurological and psychiatric symptoms, we should consider the possibility of CPM, even if the sodium level is normal.

\section{Conflicts of Interest}

No potential conflict of interest relevant to this article was reported.

\section{Author Contributions}

Conceptualization: Wei Hung Chang. Data acquisition: Yu Chia Liu. Formal analysis: Yu Chia Liu. Supervision: Po See Chen. Writing - original draft: Yu Chia Liu. Writingreview \& editing: Yen Kuang Yang, Po See Chen, Wei Hung Chang.

\section{ORCID}

Yu Chia Liu https://orcid.org/0000-0002-0079-4715 Yen Kuang Yang Po See Chen https://orcid.org/0000-0001-9355-9636 Wei Hung Chang https://orcid.org/0000-0003-4963-578X https://orcid.org/0000-0002-5964-106X

\section{REFERENCES}

1. Lampl C, Yazdi K. Central pontine myelinolysis. Eur Neurol 2002;47:3-10.

2. Ashrafian H, Davey P. A review of the causes of central pontine myelinosis: yet another apoptotic illness? Eur I Neurol 2001;8:103-109.

3. Martin RJ. Central pontine and extrapontine myelinolysis: the osmotic demyelination syndromes. J Neurol Neurosurg Psychiatry 2004;75 Supp/ 3(Supp/ 3):iii22-iii28.

4. Liu J, Dong Q, Lu X, Sun J, Zhang L, Wang M, et al. Exploration of major cognitive deficits in medication-free patients with major depressive disorder. Front Psychiatry 2019;10:836.

5. Bose P, Kunnacherry A, Maliakal P. Central pontine myelino/ysis without hyponatraemia. J R Coll Physicians Edinb 2011; 41:211-214.

6. Kilinç M, Benli US, Can U. Osmotic myelinolysis in a normonatremic patient. Acta Neurol Belg 2002;102:87-89.

7. Han MJ, Kim DH, Kim YH, Yang IM, Park JH, Hong MK. A case of osmotic demyelination presenting with severe hypernatremia. Electrolyte Blood Press 2015;13:30-36.

8. Shah MK, Mandayam S, Adrogué HJ. Osmotic demyelination unrelated to hyponatremia. Am J Kidney Dis 2018;71:436440.

9. De Picker L, Van Den Eede F, Dumont G, Moorkens G, Sabbe BG. Antidepressants and the risk of hyponatremia: a class-byclass review of literature. Psychosomatics 2014;55:536-547.

10. Twardowschy CA, Bertolucci CB, Gracia Cde M. Pontine and extrapontine osmotic myelinolysis after the syndrome of inappropriate secretion of antidiuretic hormone (SIADH) associated with fluoxetine: case report. Arq Neuropsiquiatr 2007; 65:858-864.

11. Lupato A, Fazio P, Fainardi E, Cesnik E, Casetta I, Granieri E. A case of asymptomatic pontine myelinolysis. Neurol SCi 2010;31:361-364. 